\title{
Evaluating a Human-Robot Interface for Exploration Missions
}

\author{
Alberto Valero* Paloma de la Puente ${ }^{* *}$ \\ Diego Rodríguez-Losada*** \\ * Univ. Politécnica de Madrid (UPM) (e-mail: avalero@etsii.upm.es) \\ ** UPM (e-mail: paloma.delapuente@upm.es) \\ *** UPM (e-mail: diego.rlosada@upm.es)
}

\begin{abstract}
The research reported in this paper concerns the design, implementation, and experimental evaluation of a Human-Robot Interface for stationary remote operators, implemented for a PC computer. The GUI design and functionality is described. An Autonomy Management Model has been implemented and explained. We have conducted user evaluation, making two set of experiments, that will be described and the resulting data analyzed. The conclusions give an insight on the most important usability concerns, regarding the operator situational awareness. The scalability of the interface is also experimentally studied.
\end{abstract}

Keywords: Human-Factors, Human-Machine Interface, Mobile robots, User Experiments, Autonomy Management, Situational Awareness

\section{INTRODUCTION}

\begin{abstract}
Mobile robots are increasingly becoming an aid to humans in accomplishing dangerous tasks. Examples of such tasks include search and rescue missions, military missions, surveillance, scheduled operations (such as checking the reactor of a nuclear plant for radiation), and so forth. The advantage of using robots in such situations is that they accomplish highrisk tasks without exposing humans to danger: robots go where humans fear to tread. Teaming humans and robots requires a Human-Robot Interaction (HRI) system. The purpose of such a system is to permit humans and robots to cooperate in order to accomplish cognitively demanding tasks within a spectrum of possibilities ranging between full autonomy and full teleoperation. A good HRI system should improve the accomplishment level of a task by drawing on the capabilities of both the artificial and the human agent. To this end, the operators should be able to control and/or supervise the operations of the robots through a Graphical User Interface (GUI). The GUI should provide these operators with the Situational Awareness (SA) and command capabilities required for an effective operation.
\end{abstract}

A commonly accepted definition of SA in connection with HRI is "the understanding that the human has of the location, activities, status, and surroundings of the robot" (Yanco et al. (2003)). Two aspects of SA are important for a GUI design: location awareness, defined as a mapbased concept allowing the user to locate the robot in the scenario, and surroundings awareness, which pertains to obstacle avoidance and allows the user to recognize the immediate surroundings of the robot. Spatial Cognition studies have shown that a navigator (in our case, the remote operator) having access to both of the above-mentioned perspectives exhibits more accurate performance.
Clearly, when the operator is not physically in the navigation scenario, the interface must enhance his spatial cognitive abilities by offering multilevel information about the environment (route and survey knowledge). Complex interfaces can provide different perspectives on the environment (a bird's eye view or a first-person view). Such information allows an operator looking at a GUI to have access to more than one perspective at the same time. These spatial cognitive aspects should be taken in consideration when designing a human-robot interface for remote teleoperation.

The research reported in this paper concerns the design, implementation, and experimental evaluation of a GUI for stationary remote operators, implemented for a PC computer. The GUI was designed taking account of the aspects common to both Human-Robot Interaction and and Human-Computer Interaction. As an initial design, they took the GUIs present in the literature, mainly those described in Yanco et al. (2007). The initial design, reported in this paper, then evolved through experimental evaluation.

\section{INTERFACE DESCRIPTION}

Our interface is designed for controlling multiple robots in structured and partially unstructured environments. Its goal is the ability to control a robot team in such situations, which mainly involve exploration, navigation and mapping issues. The interface design is principally concerned with providing surroundings and location situational awareness (Scholtz et al. (2004)Yanco and Drury (2004)) to an operator who must control a team of robots. Its main purpose is to enhance the operators teleoperation of a robot by affording him a comprehensive global overview of the whole team. Our concern was that the global information should be visible at all times on the screen in order to enable monitoring the entire robot 


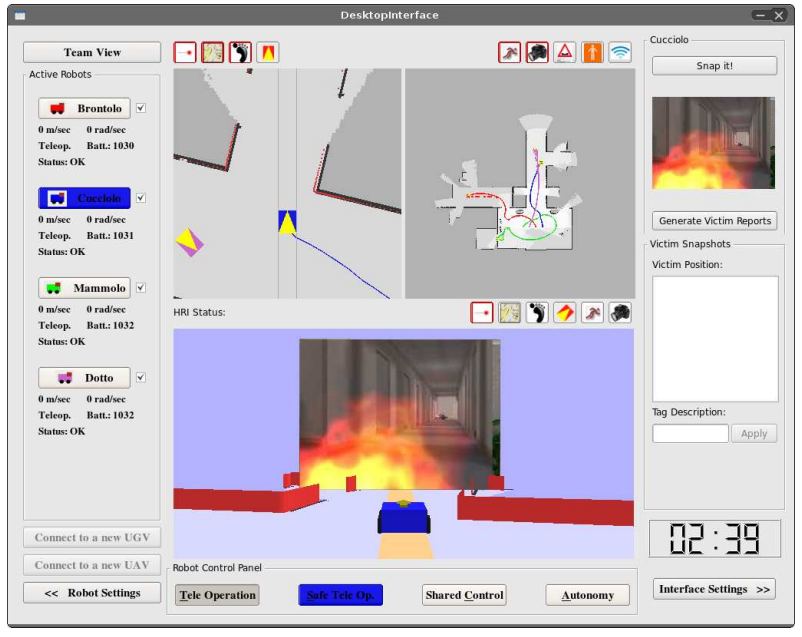

Fig. 1. Interface: Complex View

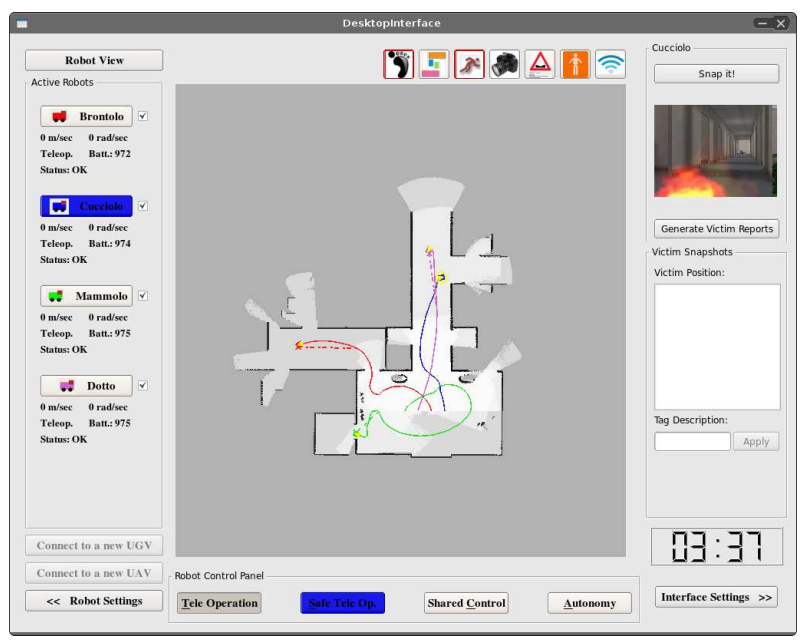

Fig. 2. Desktop Interface: Team View

team while controlling each individual robot. We will now describe the display of the data.

\subsection{Providing Situational Awareness}

Our interface prototype was mainly inspired by the INL interface as it is reported in Bruemmer et al. (2005) and the UMass-Lowell interface described in Baker et al. (2004).

The interface is shown in Figures 1 (Complex View) and 2 (Team View). The Complex View includes allocentric and egocentric views of the scenario. It consists of three views: a Local View of the Map; a Global View of the Map giving a birds-eye view of the explored area, and a pseudo-3D View giving a first person perspective on it. The Team View shows the global view of the map using the whole display.

2.1.0.1. Local View The local view of the map is designed to provide a precise surroundings awareness of the robot through an allocentric point of view defined by the robots position (this position is always fixed in the interface, and only the robots orientation changes). The operator can see the robot inside the constructed map. The operator can zoom the view in and out choosing the level of detail he desires. The robot is represented by a solid rectangle, and its direction by a solid triangle. Each robot is marked with a different color to help the operator identify which robot is operating. The map may be north-oriented or robot-oriented, depending on the operator need.

2.1.0.2. Map View The global map view provides a birds-eye perspective. In the global view of the map, all the individual maps are fused into one. All the robots are indicated inside the map. The path the robot has followed is also traced in the color of the robot. This view provides a precise location awareness of the whole team. The map is resized as the area it covers expands, which ensures that the map always fits in the display. The operator can select rectangular areas to zoom.

In both the local and map view are designed the target point each robot is trying to reach (in autonomy or shared control) and the path that will try to follow in order to reach the target point.

2.1.0.3. Pseudo-3D View The pseudo $3 \mathrm{~d}$ view of the environment is designed to provide surroundings awareness of the robot through a point of view defined in terms of robot position. A revolving arrow on the top of the robot indicates the direction of the robot. The operator can shift the perspective, either behind and above the robot or "in the place" of the robot, and thus has a first person point of view of the situation. Conversely to the INL display, the operator can choose to view either the constructed map or the laser sensor readings, which are computed to appear as a single continuous reading. This is especially useful in two situations: 1) When the map is mistaken, the operator can choose the laser view, which shows the correct position of obstacles in front of the robot. 2) In very narrow spaces, the map may not be precise enough to provide an adequate surroundings awareness, while the laser is far more exact.

This display design covers the two types of situational awareness required by an operator for controlling the robot. Surroundings awareness is provided in a precise way by the local view and the $3 \mathrm{~d}$ view, which show both laser and map readings, thereby avoiding the problem of wrongly constructed maps. Location awareness is provided by the global map view or the $3 \mathrm{~d}$ view; either way, the field of vision is set above the environment. It has been shown in Nielsen and Goodrich (2006) that an operator having several displays (in the case examined, video and map) would pay attention only to one of them. We agree with this finding, but, if he has more than one display to choose from, the operator can switch from one to another according to his needs. It seems clear that none of the views described is the most appropriate for all situations. Furthermore, this design, as should be clear, supports the control and supervision of a team of robots, as it includes robot-attached views as well as allocentric views, thus avoiding the problem raised by INL and UMass-Lowell designs in Yanco et al. (2007). 


\subsection{Autonomy Management}

The autonomy is adjusted in a multi-layered form. The following list describes the different autonomy levels, beginning with full autonomy and ending with pure teleoperation. This structure is inspired by Sheridan (2002).

(1) The system proposes a target point;

(2) if there is no input from the operator the system proposes a path to reach it, or

(3) the operator sets a target point and the system proposes a path to reach it;

(4) if the operator does not change the path the system follows the path, or

(5) the operator sets a path and the system follows it;

(6) The operator sets a control speed and jog and the system adjust the real speed and jog to avoid collisions.

(7) The operator sets the real speed and jog.

From this list we can see that the operator has five command possibilities possibilities: set a target point (or sequence of target points), set a path, set a control speed and jog, set the real speed and jog. Depending on the operation mode of the robot: Tele-Operation, Safe TeleOperation, Shared Control or Autonomy, the system will react differently to each input. The possibilities are:

2.2.0.4. Tele-Operation and Safe Tele-Operation. If the operator sets a target point or path, the robot changes to shared control.

2.2.0.5. Shared Control. In shared control mode, the system tries to reach the target point, or sequence of target points, set by the operator. This is done by means of two mechanisms: the calculation of a path and the control of robot motion to follow that path. The operator can give the following commands.

- Speed commands: The system remains in Shared Control, but while the operator continues sending speed commands, the robot's motion is not controlled by the system. When the operator stops sending commands, the system retakes motion control. This is very efficient when the robot is following a path and remains stalled, because the operator can get the robot out of its stalled condition without modifying its former task. As soon as the operator releases the control, the robot continues the previous task.

- The operator sets a path, the robot follows that path, and it then calculates a new path to the former target point, if there was one. In this way, if the operator disagrees with the path proposed by the system, he can modify it, and the robot adjusts its proposed path to the path set by the operator.

Figure 3 show an example of how this works. The system is in Shared Control Mode. The operator modifies the path proposed by the system, deactivating temporarily the Path-Planning Layer. Once the robot has completed the path, this layer will re-activate.

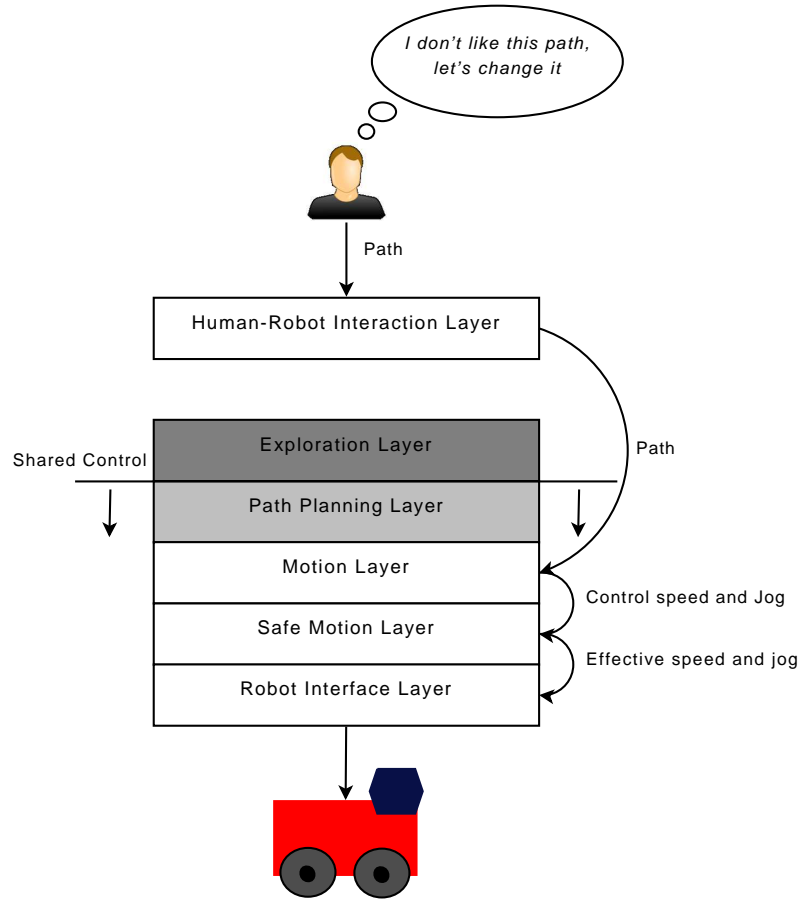

Fig. 3. Shared Mode. Operator sets a path

2.2.0.6. Autonomy. In full autonomy, the system chooses the most suitable target point in order to accomplish the mission. The operator may send the following commands:

- Speed, Jog and Path, as in Shared Mode. The system gives the suitable control to the operator, but remains in autonomy, so that, when the operator stops commanding, the system keeps on working in autonomy. Like shared mode, this mode is suitable in situations in which the operator does not approve the actions taken by the system.

- The operator sets a target point or sequence of target points. The system tries to reach them, and, once they have all been reached, it keeps on exploring autonomously.

- The operator sets a desired path. The system tries to follow it, once it has completed the task, and if there are not target points, it keeps on exploring autonomously (if there is a desired path and a sequence of target points it will follow firstly the desired path, and afterwards it will try to reach the target points).

Each layer is in charge of performing an action. The more layers are working, the higher the autonomy level is. Thus, if all the layers are running, the system is working in full autonomy, while, if none of them is working, the operator has full control of the robot. The operator can command at every level of the layered system, substituting the actions of the higher layers. It is important to notice that this does not require him to change the operation mode. For example: The system may be operating in "Autonomy Mode," so that, by default, all the layers are running, but at any moment the operator can command at any level, while the system remains in "Autonomy Mode." At the same time, every layer provides feed-back indicating whether or not it has performed its respective task, so the operator has correct system state awareness and knows at which level he must act. 
This autonomy management is thought to provide the operator with two advantages:

- Fast recovery from navigation and exploration errors or bad performance. The operator can act at the error level, without re-configuring the robot task or changing the operation mode.

- Long-term commands and more granulated autonomy levels. The operator can set a sequence of target points, giving the robot a longer-term task. Moreover, since he can set the path, something that was not possible in previous version, he can send the robot along safe paths.

In this way, the system takes more advantage of the operator's expertise.

\section{INTERFACE DESIGN EVALUATION}

The first battery of experiments were designed to measure the usability of the interface developed.

\subsection{Experiment Design and Procedure}

Students were enrolled and a disaster scenario was simulated on the playground of the University. The experiments involved twenty-four subjects, nineteen undergraduates and five $\mathrm{PhD}$ candidates ranging in age between 20 and 30 and distributed among four females and twenty males. No participant had previous experience of either of the two interface prototypes. All the subjects went through the experiments in the same order, which ensured that no one had more experience than the others. Every subject went through a twenty-minute training program to acquire a basic knowledge of the functionalities provided by the interfaces. After the training, they ran through the experiments in order. Each subject had a single trial.

Experiments were conducted with the a P2AT robot in both indoor and outdoor scenarios. Subjects were asked to explore a maze and navigate along a path, approximately 15 meters in length, made up of narrow spaces and clustered areas.

We asked users to "think aloud" during the task, as we wished afterwards to apply the LASSO evaluation technique (Drury et al. (2007)).

We sought to measure whether the integration of several displays providing allocentric and egocentric information would meet our expectations. We enumerate here the main usability concerns expressed by the majority of subjects. We will list their positive and negative usability remarks regarding operator situational awareness.

\subsection{Results}

\section{Surroundings Awareness}

\section{Negative Remarks}

- 3D View: i) When the perspective in the 3D view is behind the robot correct perception of the distance to the obstacles is compromised, ii) rear obstacles are not visualized if they are not on the map, which may therefore be imprecise and confusing, iii) the map may become too imprecise to guide the robot through very narrow spaces, iv) the elevated map sometimes hides the robot, v) when the laser and the map do not coincide exactly, the map may become a source of confusion, and vi) when the camera pans, the video may be difficult to see.

- Local Map View: i) Usually the operator lost location awareness (regarding the orientation) when the view is robot-oriented, ii) if the map is imprecise it is not suited for navigating in narrow spaces.

\section{Positive Remarks}

- 3D View: i) The laser view helps to identify with precision where the real obstacles are, ii) the laser view shows moving obstacles that do not appear on the map, and iii) Looking at the 3D view from above with the laser view, one can navigate easily through narrow spaces.

- Local Map View: If the map is right, this view is very useful for narrow spaces, because one can zoom as much as one wishes, knowing precisely where rear and front obstacles are.

\section{Location Awareness Negative Remarks}

- 3D View As the robot is always facing up, one can go south while thinking that one is going north.

- Global Map View: i) The map is too small when the explored area is very big and it is very hard to localize the robot, and ii) the path can become a source of confusion when the robot has been moving for a long time.

\section{Positive Remarks}

- Local Map View: i) One can see the orientation of the robot, and ii) when the global map is too big for the global view, one can zoom out the local view and have a good idea where the robot is.

- Global Map View: i) One knows where every robot is, and ii) the design of the path indicates where the robot has been previously.

All these considerations confirmed that integrating the map-centered view of INL interface and the VideoCentered proposal of UMass-Lowell interface (see Yanco et al. (2007)) is more suited for general exploration missions, as any of the perspectives is more required depending on the scenario and task.

\section{INTERFACE SCALABILITY EVALUATION}

As we said at the beginning, an effective operation of multiple robots requires that an operator distribute his operating time among the robots, so that he can supervise the whole team and intervene whenever this is required. The experiment we are presenting here aimed to evaluate the scalability of the interface.

\subsection{Experiment Design and Procedure}

The experiment was organized as a competition. Participants were given the incentive of two prizes (an iPod and an HTC cell phone). They were scored following the RoboCup Virtual Rescue Robots League criteria: area 


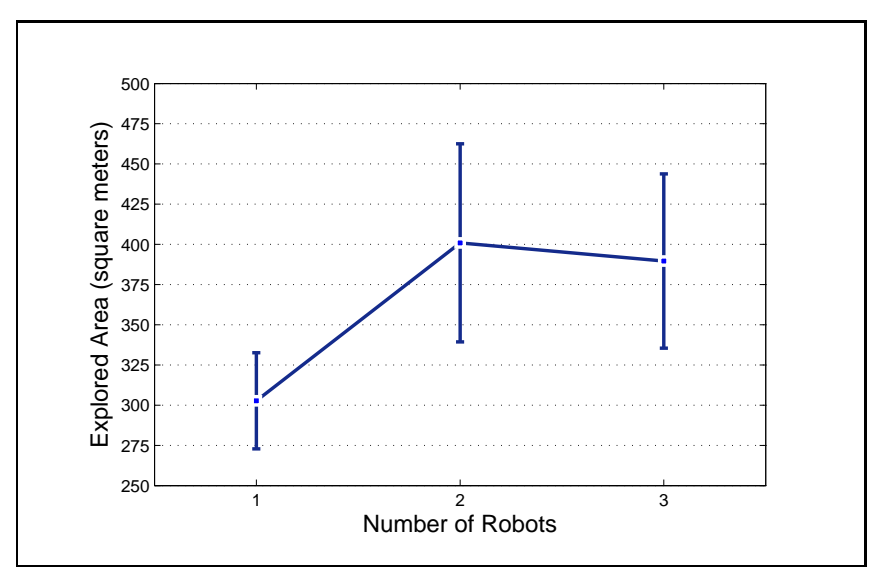

Fig. 4. Explored Area - Confidence Intervals (95\%)

covered and cleared and number of victims found. Fortyfive subjects (forty-one males, four females) participated in the competition. All of them were either master's students or Ph.D. candidates. The subjects were randomly divided into three groups. Each subject in a given group had to accomplish a Search and Rescue Mission while controlling a team of robots (number of robots $\in[1-3]$ ). Every subject went through a forty-minute training program to acquire a basic knowledge of the functionalities provided by the interface. The training scenario was taken from the RoboCup Competition and was similar in difficulty to the scenarios used for the experiments.

The participants were asked to explore an unknown office building (indoor scenario) in search of victims. Subjects were given 20 minutes.

\subsection{Data analysis}

The focus of our analysis is the influence of the factor number of robots $\in\{1,2,3\}$. Though we analysed more of the data collected, here we present only the explored area, as a measure of the operator performance. The area explored is measured in square meters. The number of subjects in each group is 15. A one-way ANOVA was carried out to study if there were any significant differences. As the p-value was less than 0.05 , we saw that the area explored differs significantly from group to group. We then calculated the confidence intervals for a $95 \%$ value in order to get a handle on these. Confidence intervals can be seen in Figure 4

The analysis of the area explored reveals that the explored area is greater for 2 and 3 robots than for 1 robot. The differences are statistically significant. For 2 and 3 robots, there is no statistical difference (Figure 4).

\section{DESIGN EVOLUTION}

The test results presented in the previous section were satisfied with the 2D maps, the local display providing surroundings situational awareness, and the global map display (including the global map view) giving a comprehensive view of the whole robot team. The majority of their comments concerned the $3 \mathrm{D}$ view.

Furthermore, subjects noted that when the map is completely mistaken (this happened often in the outdoor run,

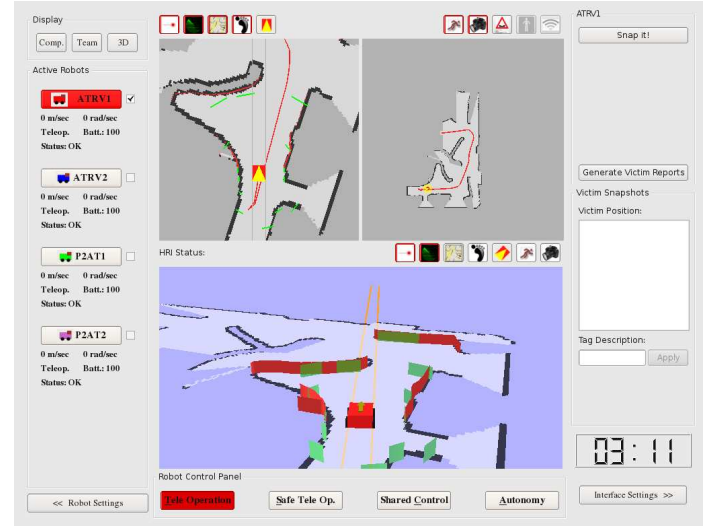

Fig. 5. Interface with Sonar Readings (green) and Laser Readings (red)

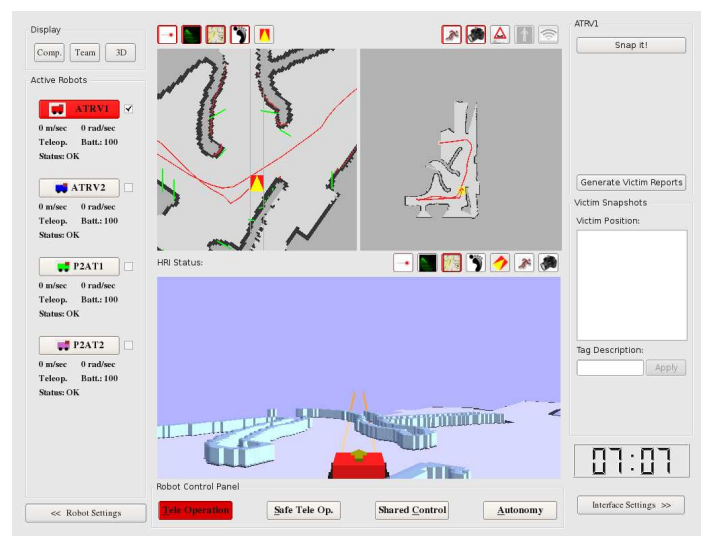

Fig. 6. Interface with the 3D Map

as the open spaces caused the scan-matching algorithm to fail) they have to rely completely on the video and the laser, while the 2D maps are useless.

With these issues in mind, we made the following changes in the $3 \mathrm{D}$ view:

- We included the sonar readings coming from the robot, which affords a real-time position of rear obstacles. See Figure 5;

- We drew the map flat and not elevated, so that it does not hide the robot and is not confused with the robot.

- The point of view can be set in such a way that the image always remains in the front part of the display, while the robot and the environment are rotated according to the pan of the camera. See Figure 7.

Furthermore, we added a new display to the two existing ones: "robot view" and "team view." The new display shows only the 3D view, which gives the operator more viewing room when he wishes to see only the video and the laser and sonar readings. See Figure 8.

\section{CONCLUSIONS AND FURTHER WORK}

In this paper we have evaluated experimentally the usability of a human-robot interface and the scalability in terms of number of robot that an operator can control simultaneously, getting that the optimal number is two robots. 


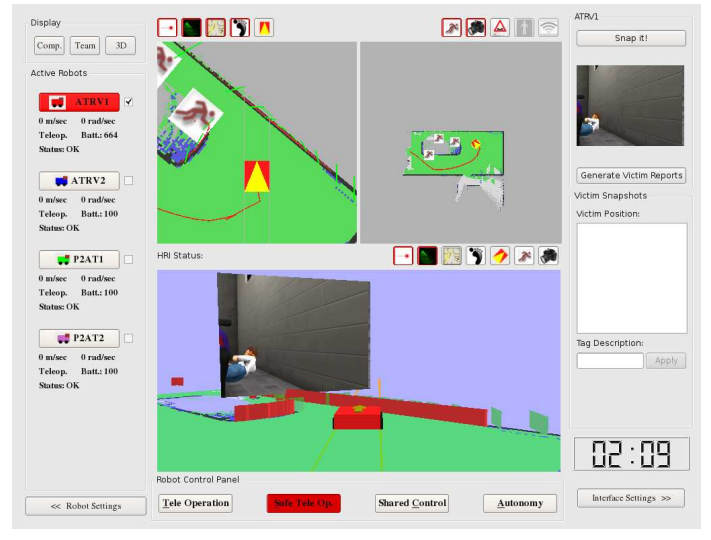

(a) Robot-Centred View

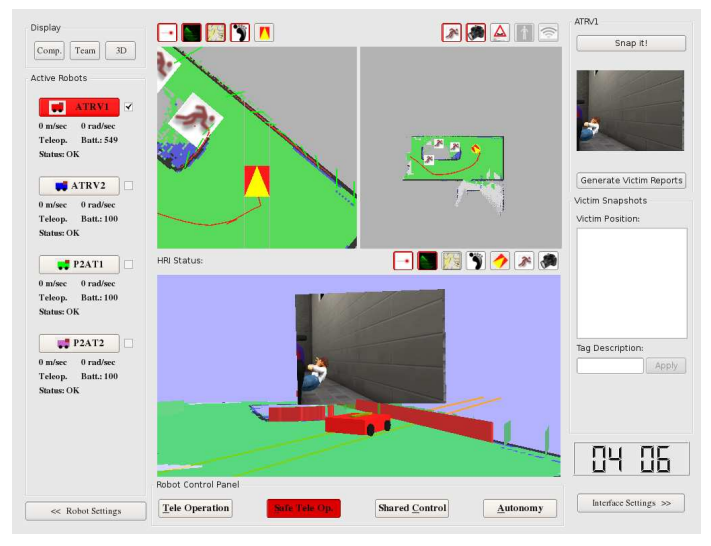

(b) Camera-Centred View

Fig. 7. Different Display Angles of the Video Feed-back

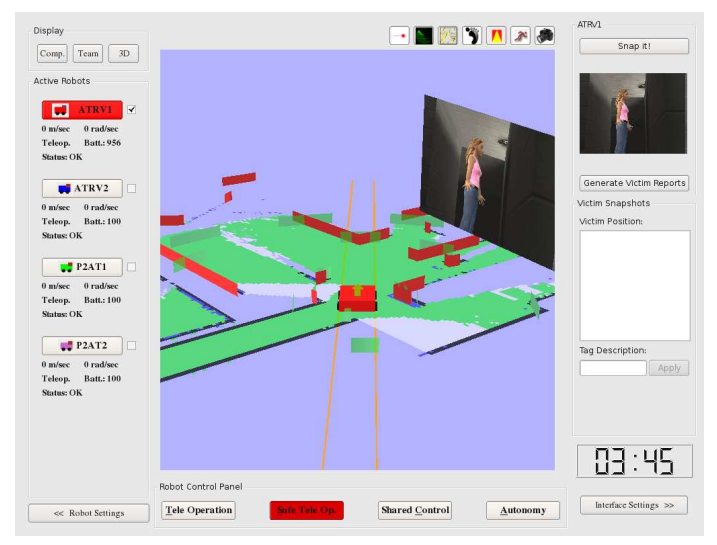

Fig. 8. Interface with the 3D View Display

The evaluation of the this version of the interface has lead to the next version, in which a configurable autonomy mode was implemented. While previously autonomous exploration was defined completely within the robot's software, now the operator can parametrize it, which allows him to configure exploration policies for the robotic team. The functionalities included are:

- Set compulsory and forbidden destination areas,

- define navigable and non-navigable regions,

- set preferred exploration directions, and

- set a final destination that the robot should eventually reach if it can.
This version of the interface was used in the last RoboCup Edition (2009, Austria), in the Rescue Simulation League. It received a Technical Award for the most innovative interface. Further work will include the conducting of user experiments to evaluate the improvements brought by actual version. We hypothesize that, with the new functionalities offered in Autonomy Mode we will increase the optimal team size to three or even four robots.

\section{REFERENCES}

Baker, M., Casey, R., Keyes, B., and Yanco, H.A. (2004). Improved interfaces for human-robot interaction in urban search and rescue. In Proceedings of the IEEE International Conference on Systems, Man \& Cybernetics: The Hague, Netherlands, 10-13 October 2004, 29602965.

Bruemmer, D.J., Few, D.A., Walton, M.C., Boring, R.L., Marble, J.L., Nielsen, C.W., and Garner, J. (2005). "turn off the television!" : Real-world robotic exploration experiments with a virtual 3-d display. In 38th Hawaii International Conference on System Sciences.

Drury, J.L., Keyes, B., and Yanco, H.A. (2007). Lassoing hri: analyzing situation awareness in map-centric and video-centric interfaces. In Proceedings of the Second ACM SIGCHI/SIGART Conference on Human-Robot Interaction, 279 - 286.

Nielsen, C.W. and Goodrich, M.A. (2006). Comparing the usefulness of video and map information in navigation tasks. In Proceedings of the 1st ACM SIGCHI/SIGART Conference on Human-Robot Interaction, HRI 2006, 95101.

Scholtz, J., Young, J., Drury, J.L., and Yanco, H.A. (2004). Evaluation of human-robot interaction awareness in search and rescue. In Robotics and Automation, 2004. Proceedings ICRA'04, volume 3, 2327 - 2332. IEEE.

Sheridan, T.B. (2002). Humans and Automation. System Engineering and Management. John Wiley and Sons, Inc.

Yanco, H.A. and Drury, J.L. (2004). "where am i?" acquiring situation awareness using a remote robot platform. In Proceedings of the IEEE International Conference on Systems, Man $\&$ Cybernetics: The Hague, Netherlands, 10-13 October 2004, 2835-2840.

Yanco, H.A., Drury, J.L., and Scholtz, J. (2003). Awareness in human-robot interactions. In Proceedings of the IEEE Conference on Systems, Man and Cybernetics, Washington, DC, October 2003.

Yanco, H.A., Keyes, B., Drury, J.L., Nielsen, C.W., Few, D.A., and Bruemmer, D.J. (2007). Evolving interface design for robot search tasks. Journal on Field Robotics, 24(8-9), 779-799. 